\title{
A patient with tremor, part 1: making the diagnosis
}

\author{
Hrishikesh Kumar MD DM, Mandar Jog MD
}

\section{The case}

A 58-year-old woman presented with shaking of her left hand that had started insidiously and progressively worsened over the past few years. She also complained of stiffness in her left arm. The shaking was more pronounced when she rested her hand. Although the shaking decreased when she outstretched her arms, it persisted when she walked (Appendix 1, video available at www.cmaj.ca/lookup/suppl /doi:10.1503/cmaj.091782/-/DC1).

$\mathrm{T}$ remor, defined as rhythmic, involuntary oscillations of a body part involving alternate contraction of agonistic and antagonistic muscle groups, is commonly seen in community practice. About $5 \%$ of people over 40 years of age are affected by tremor. ${ }^{1}$ Tremor can be a manifestation of a wide variety of neurologic and nonneurologic disorders. ${ }^{2}$ Essential tremor, tremor in Parkinson disease and enhanced physiologic tremor are the commonest varieties, but a number of other causes should be considered. A methodic approach to the classification and diagnosis of tremor is therefore needed to simplify and expedite the management of this condition, saving time and resources.

This article aims to present a simple stepwise approach for the diagnosis of tremor. In part 2 of this series, we will discuss some classic presentations paired with short video clips that highlight the subtypes of tremor and guide the diagnostic and therapeutic discussions. The presented approach involves identification and description of the pattern of tremor, phenomenologic classification and then determination of cause. This standard method of evaluating a patient with abnormal movements has been endorsed by The Movement Disorder Society's consensus statement on tremor. ${ }^{3}$

\section{Is this tremor?}

The first step in the evaluation of a patient presenting with "shaking" is to determine whether the abnormal movements are really tremor, because various movements can be erroneously described as "shaking" or "tremor." Myoclonus, epilepsia partialis continua, clonus and stereotypy may be mistaken as tremor.

A rhythmic character of the movement is the key feature that differentiates tremor from other mimics. Myoclonus is differentiated from tremor by its nonrhythmicity and jerky character. Epilepsia partialis continua can be rhythmic, but the movements do not change with alteration in position or activity. Clonus may be mistaken as tremor because it occurs predominantly at joints. But, unlike tremor, clonus increases in amplitude and force with passive stretching of the muscles. Other hyperkinetic disorders such as dystonia, tic and chorea are less likely to be mistaken as tremor because of their distinctive nonrhythmic character. However, awareness of these conditions is important, especially because they can coexist with tremor. Key features of common hyperkinetic conditions are provided in Table $1{ }^{4}$

\section{Objective at this stage}

We should be able to recognize whether the patient has tremor or some other hyperkinetic movement disorder. It is also important to know whether the tremor is isolated or associated with other symptoms.

\section{- KeY POINTS}

- Tremor is a common symptom in primary care and has a wide differential diagnosis.

- It is defined as rhythmic, involuntary oscillations of a body part involving alternate contraction of agonistic and antagonistic muscle groups.

- A methodic approach is needed to determine the underlying etiology and to formulate a plan for treatment.

- One approach involves identification and description of the tremor, phenomenologic classification and determination of the cause using data from the patient history and examination.
Competing interests: Mandar Jog has served on the boards of Allergan, Inc., Biovail Corp., Novartis and Teva Pharmaceutical Industries Ltd. He has received payment for consulting or lecturing from Allergan, Inc., Teva

Pharmaceutical Industries Ltd., Biovail Corp. and

Novartis. His institution has received funding from Canadian Institutes of Health Research, the Natural Sciences and Engineering Research Council of Canada and Parkinson Society Canada

None declared by Hrishikesh Kumar.

This article has been peer reviewed.

Correspondence to: Dr. Hrishikesh Kumar, rishi_medicine@yahoo.com

CMAJ 2011. DOI:10.1503 /cmaj.091782 
Table 1: Hyperkinetic disorders and their key diagnostic features ${ }^{4}$

\section{Hyperkinetic} disorder

Key features

\begin{tabular}{|ll|}
\hline Tremor & Rhythmic, involuntary oscillations of a body part \\
\hline Clonus & $\begin{array}{l}\text { Rhythmic oscillations around a joint in response to sudden } \\
\text { passive contraction }\end{array}$ \\
\hline Stereotypy & Repeated and continuous complex motor acts \\
\hline Myoclonus & Very rapid, jerky, shock-like movements \\
\hline Dystonia & $\begin{array}{l}\text { Sustained muscular contractions frequently causing twisting } \\
\text { and abnormal posturing of a body part }\end{array}$ \\
\hline Tic & $\begin{array}{l}\text { Nonrhythmic, nonstereotyped, semipurposive, relatively } \\
\text { slow movements that flow from one part of the body to } \\
\text { another }\end{array}$ \\
\hline & $\begin{array}{l}\text { Brief, nonrhythmic, rapid movements that are usually } \\
\text { orofacial but may involve the voice or any part of the body; } \\
\text { presence of urge to perform; can be suppressed voluntarily } \\
\text { for a short period, with rebound increase after the period } \\
\text { of suppression }\end{array}$ \\
\hline
\end{tabular}

\section{Box 1: Clinical classification of tremor, based on phenomenology}

Resting tremor: The tremor is present in the body part that is not voluntarily activated, and the body part in question is completely supported against gravity. Observation of the patient resting on a couch or supporting the limb on the armrest of a chair is the best way to look for resting tremor.

Action tremor: The tremor is seen during voluntary activity of the muscles. Having the patient perform the spiral test (patient is asked to draw a spiral inside another spiral), join two points between closely spaced straight lines and pour water from one glass to another are methods to objectively assess the severity of action tremor. The action tremor is further classified as postural and kinetic tremor.

- Postural tremor: The tremor appears with voluntary maintenance of an antigravity position. Having the patient outstretch the arms for 10-15 seconds, keep a loose sheet of paper on the hand or add some weight in the hands (e.g., asking the patient to hold a cup filled with water) will make the tremor more obvious.

- Kinetic tremor: This includes any tremor that is present during voluntary movements. Various reaching movements (e.g., nose-finger-nose and knee-heel-shin tests) are performed as part of the clinical examination to detect kinetic tremor. Kinetic tremor is subdivided into the following categories:

a. Simple kinetic tremor: The tremor is present during all voluntary movements that are not targeted, as in repeated flexion-extension of the elbow or pronation-supination of the forearm. No increase in amplitude in the terminal phase is noted in simple kinetic tremor.

b. Intention tremor: The tremor is present during target-directed movements. Tremor amplitude increases as the hand or body part approaches the visually directed target. Use of the term "intention tremor" has been criticized because it is not the mental intention that aggravates the tremor but the performance of target-directed movements. However, the term is deeply engrained in neurologic language.

c. Task-specific tremor: The tremor appears or becomes exacerbated during a specific activity (e.g., primary writing tremor and musician's tremor). Isolated vocal tremor is a type of action tremor and can be brought out by asking the patient to hold a note as long as possible.

d. Isometric tremor: The tremor occurs as a result of contraction of muscles against a rigid stationary object.

\section{What are the characteristics of the tremor?}

The next step is to describe the tremor in a way that gives a clear picture of its characteristics. A thorough method of description, applicable to all movement disorders, includes distribution, amplitude, frequency, presence or absence during rest, effect of activities on the tremor, changes with distraction, effect of manoeuvres to bring out the tremor (if the tremor is not visible) and entrainment. Entrainment is a clinical finding observed in patients with psychogenic tremor and is described below.

The patient is asked to do rapid alternating movements of the limb that is not in question (opening and closing of the fist, pronation and supination of the forearm, and foot tap on the floor) and the limb in question is closely observed for the following:

- subtle tremor becomes more obvious, as occurs with resting tremor in Parkinson disease;

- the tremor that is already present disappears during movement of other limbs (distractibility, which occurs with psychogenic tremor) $;^{5}$

- the tremor that is already present changes its rhythm and takes on the rhythm of the voluntarily moving limb (phenomenon known as "entrainment," seen in psychogenic tremor). ${ }^{5}$

\section{Objective at this stage}

We should be able to clearly describe the tremor under various headings, so that a description can give a mental picture of the characteristics of the tremor to the listener. The tremor seen in the video in Appendix 1 would be described as rhythmic, involuntary oscillations of the left upper limb, predominantly involving the wrist and distal portions of the fingers, present during rest with decrease during activities, slow in frequency, moderate to severe in amplitude, increased by distracting movements of other limbs and having no entrainment.

\section{How should the tremor be classified?}

A thorough description of the tremor allows for phenomenologic classification. The system of classification outlined in Box 1 was proposed by the Movement Disorder Society's ad hoc scientific committee on tremor in $1998 .^{3}$ The proposed classification was based on earlier work and has been widely used in subsequent reviews and research papers. ${ }^{1,2,6-8}$ 
Box 2: Strategies to determine the cause of tremor ${ }^{3,9,10}$

\section{Phenomenologic classification}

- Resting tremor: tremor in Parkinson disease

- Postural tremor: enhanced physiologic tremor, essential tremor with or without vocal tremor, psychogenic tremor

- Tremor with goal-directed movements (intention tremor): cerebellar tremor, adverse effect from lithium (rarely), chronic intake of alcohol

- Task specific: primary writing tremor, musician's tremor, isolated vocal tremor

- Special tremor: Holmes tremor, palatal tremor, orthostatic tremor, dystonic tremor

\section{Frequency and amplitude of tremor}

- Frequency: Tremors in different conditions tend to involve a characteristic range of frequency. It is difficult to determine the exact frequency clinically, but one can ascertain whether tremor is slow, moderate or fast in frequency and use this information as a clue to diagnosis (Table 2).,11

- Amplitude: Amplitude is not always helpful because it can vary with time in the same patient. But, as a general rule, orthostatic tremor and enhanced physiologic tremor are of small amplitude, essential tremor is of moderate amplitude, and Holmes tremor and parkinsonian tremor are of high amplitude.

Historical data: A thorough history and clinical examination are imperative, and the following information should be especially looked for.

- Age at onset: Essential tremor has a biphasic peak for age at presentation. The first peak appears during the early teens and the second in late adulthood. It is not uncommon for a patient with essential tremor to have a history of tremor for a few years during the teenage years and then to have a reappearance in late adulthood. ${ }^{9}$ Tremor related to Wilson disease or congenital cerebellar malformations also appears early. Tremor in patients with Parkinson disease and orthostatic tremor usually appear in later age.

- Mode of onset and progression: Acute-onset tremor may be seen in stroke (cerebellar or midbrain), multiple sclerosis and cerebellitis. Tremor in patients with cerebellar tumours and paraneoplastic cerebellar degeneration is usually subacute in onset. Progression of tremor is slow in essential tremor (over decades) and in Parkinson disease (over years).

- Symmetry: Essential tremor and drug-induced enhanced physiologic tremor are usually bilateral and symmetric. Asymmetry is the hallmark of tremor associated with Parkinson disease, but use of neuroleptics can produce similar asymmetric resting tremor. Dystonic tremor and primary writing tremor are limited to a specific body part. Tremor secondary to brain lesions (cerebellum, midbrain, thalamus) may be focal, depending on the site of the lesion.

- History of medications, substance abuse, alcoholism or alcohol withdrawal: Drugs can enhance physiologic tremor or can produce postural or resting tremor.

- Family history: Essential tremor can have an autosomal dominant pattern of inheritance, but family history is not essential for the diagnosis. A minority of patients with Parkinson disease have a positive family history. Even in these instances, severity of tremor in family members may be variable. Family history of mental retardation may be recorded in instances of fragile $\mathrm{X}$-associated tremor/ataxia syndrome. ${ }^{10}$

- Associated symptoms: Tremor is monosymptomatic in essential tremor and enhanced physiologic tremor. In instances of Parkinson disease, cerebellar disorders and Wilson disease, associated features are often present and help in diagnosis.

- General and systemic examination: A general and systemic examination, including pulse rate, enlargement of the thyroid, signs of anxiety and enlargement of the liver are helpful to indicate a specific etiology.

Neurologic examination: The following features should be especially sought during neurologic examination in patients with tremor.

- Bradykinesia: Defined as a progressive decrease in amplitude and speed on rapid alternating movements of limbs, bradykinesia suggests parkinsonism when it appears with rigidity.

- Rigidity: Defined as a feeling of resistance on passive movement of the limb, rigidity suggests parkinsonism when it appears with bradykinesia.

- Cognitive functions: Cognitive abnormalities can be seen in fragile X-associated tremor/ataxia syndrome, Wilson disease and late Parkinson disease.

- Eye signs: Nystagmus, abnormality of saccadic and pursuit movement, hyper- or hypometric saccades and square-wave jerks are signs of cerebellar disorder.

- Finger-nose test and knee-heel-shin test: These manoeuvres are done to elicit the intentional component of cerebellar tremor. 


\begin{tabular}{|ll|}
\hline $\begin{array}{l}\text { Table 2: Etiology of tremor according to } \\
\text { frequency }\end{array}$ \\
\hline Frequency & \multicolumn{1}{c|}{ Type of tremor } \\
\hline Very slow & Holmes tremor \\
\hline Slow & Tremor in Parkinson disease \\
\hline Moderate & Essential tremor \\
\hline Fast & $\begin{array}{l}\text { Physiologic and enhanced } \\
\text { physiologic tremor }\end{array}$ \\
\hline Very fast & Orthostatic tremor \\
\hline Variable & Psychogenic tremor \\
\hline
\end{tabular}

\section{Objective at this stage}

By this point, we should be able to classify the tremor into one of the subgroups mentioned in Box 1. More than one type of tremor can exist in a patient, and it is useful to determine the predominant type of tremor.

\section{What is causing the tremor?}

Phenomenologic classification facilitates clarification of the cause, with the help of clues provided by history, examination and, in some patients, investigations. Various strategies can be used, but none of them are exclusive or sufficient by themselves. Strategies to help determine the cause of the tremor (using phenomenologic classification, frequency and amplitude of tremor, historical data and findings of the neurologic examination) are outlined in Box 2.,3,9-11

\section{Objective at this stage}

On analyzing the type of tremor and with the help of various clues from the history and examination, we should be able to determine the etiology and relevant differential diagnosis. In our patient (Appendix 1), asymmetric resting tremor and the symptom of stiffness suggested idiopathic Parkinson disease. Presence of bradykinesia and rigidity on neurologic examination confirmed the diagnosis. Intake of neuroleptics (e.g., haloperidol, risperidone), tetrabenazine or antiemetics (e.g., metoclopramide, prochlorperazine) should be ruled out before Parkinson dis- ease is diagnosed because these drugs can produce similar clinical signs. Levodopa and dopamine agonists are the mainstay for the treatment of Parkinson disease.

\section{Conclusion}

Tremor is the most common involuntary movement disorder seen in clinical practice. The differential diagnosis is wide, and the diagnostic approach requires classification and analysis of data obtained from the history and examination. Using simple, observable and clinically based features, clinicians can diagnose and classify tremor. Awareness of patterns of tremor in various etiologic conditions helps in clinching the diagnosis and formulating an appropriate strategy for management. Because most types of tremor do not have an associated diagnostic test, accurate clinical judgment will determine treatment and outcome and can prevent an incorrect diagnosis that may have a substantial emotional impact.

\section{References}

1. Bain PG. Parkinsonism and related disorders. Tremor. Parkinsonism Relat Disord 2007;13(Suppl 3):S369-74.

2. Deuschl G. Differential diagnosis of tremor. J Neural Transm Suppl 1999;56:211-20.

3. Deuschl G, Bain P, Brin M. Consensus statement of the Movement Disorder Society on tremor. Ad Hoc Scientific Committee. Mov Disord 1998;13(Suppl 3):2-23.

4. Kumar A, Calne DB. Approach to the patient with a movement disorder and overview of movement disorders. In: Watts RL, Koller WC, editors. Movement disorders neurological principles and practice. New York (NY): McGraw-Hill Medical Publishing; 2004. p. 3-15.

5. Kenney C, Diamond A, Mejia N, et al. Distinguishing psychogenic and essential tremor. J Neurol Sci 2007;263:94-9.

6. Hallett M. Classification and treatment of tremor. JAMA 1991;266:1115-7.

7. Findley LJ, Koller WC. Handbook of tremor disorders. New York (NY): Marcel Dekker; 1995.

8. Raethjen J, Deuschl G. Tremor. Curr Opin Neurol 2009;22:400-5.

9. Elble RJ, Deuschl G. An update on essential tremor. Curr Neurol Neurosci Rep 2009;9:273-7.

10. Berry-Kravis E, Abrams L, Coffey SM, et al. Fragile X-associated tremor/ataxia syndrome: clinical features, genetics, and testing guidelines. Mov Disord 2007;22:2018-30.

11. Bhidayasiri R. Differential diagnosis of common tremor syndromes. Postgrad Med J 2005;81:756-62.

Affiliation: From the Movement Disorders Program, Division of Neurology, University of Western Ontario, London, Ont.

Contributors: Both authors wrote the article, revised it and approved the final version submitted for publication. 